\title{
Experimental studies on the biological effects of extracorporeal shock wave therapy on tendon models. A review of the literature
}

\author{
Vincenzo Visco ${ }^{1}$ \\ Maria Chiara Vulpiani ${ }^{2}$ \\ Maria Rosaria Torrisi ${ }^{1}$ \\ Andrea Ferretti ${ }^{2}$ \\ Antonio Pavan ${ }^{1}$ \\ Mario Vetrano ${ }^{2}$
}

\footnotetext{
1 Department of Clinical and Molecular Medicine, "Sapienza" University of Rome, Italy; Sant'Andrea Hospital, Rome, Italy

2 Department of Ortophaedics and Traumatology, "Sapienza" University of Rome, Italy; Sant'Andrea Hospital, Rome, Italy
}

\author{
Corresponding author: \\ Vincenzo Visco \\ Department of Clinical and Molecular Medicine \\ "Sapienza" University of Rome \\ Piazza Sassari 3 \\ 00161 Rome, Italy \\ E-mail: vincenzo.visco1@uniroma1.it.
}

\section{Summary}

Encouraging results using extracorporeal shock wave therapy (ESWT) for treating chronic tendinopathies were recently obtained, although the specific mechanisms by which it induces therapeutic effects remain largely unknown. In order to provide possible explications of such clinical efficacy, several reports have investigated the effects of ESWT on animal models and different kind of cultured cells. Our contribute in establishing the potential outcome of ESWT on human primary cultured tenocytes, derived from healthy compared to ruptured tendons, have supported the growing evidence that shock waves may supply faster post-injury recovery. The purpose of this review was to summarize and evaluate the available basic scientific evidences for using ESWT in tendon pathologies, suggesting possible shock waves-induced mechanisms of tissue repair.

KEY WORDS: extracorporeal shock wave therapy, ESWT, tendon.

\section{Introduction}

In the last decade, focused ESWT has been used in the treatment of tendinopathies and orthopaedic pathologies. Despite its success in clinical application for treating soft tissues and orthopaedic disorders, the biological mechanisms of ESWT are not completely elucidated, although there is an increasing confidence on a probable acceleration of the healing process by angiogenesis enhancement ${ }^{1-3}$.

Several authors proposed that its effectiveness could be ascribed to the transduction of the acoustic shock wave signal into biological signals which results in cell proliferation and/or differentiation by a mechanotransduction process ${ }^{4}$. At the beginning, to provide an interpretation of the clinical benefits of ESWT on chronic tendinopathies, earlier reports evaluated shock waves effects using models of animal-derived primary cultures ${ }^{5-7}$ and/or cell lines ${ }^{8}$, because the low cellularity of tendon limited the efficiency of performing primary cultures of human tenocytes ${ }^{9}$. Unfortunately, the results obtained on animal models do not fully apply to human conditions ${ }^{10}$. However, some cultures of human tenocytes have been more recently established also by our study group and used for better understanding the cellular and molecular mechanisms involved in tendon degeneration and repair ${ }^{11,12}$. Here we suggest some clues for better reading the ESWT-mediated efficacy in the treatment of human chronic tendinopathies.

\section{Experimental studies on animal models}

The first reports on the ESWT effects on tendon tissue were achieved on animal models to determine if shock waves treatment parameters were safe or dangerous for target tissues. Rompe et al. (1998) carried out a sonographic and histological study ${ }^{13}$, with the aim of determining the outcomes of applying different ESWT energy intensities on rabbit Achilles tendon. Dose-dependent increases in anteroposterior diameter of the tendon and formation of paratendinous fluid with histopathological changes (presence of degenerative signs and inflammatory cells, increased numbers of capillaries, oedema and fibrosis in the paratenon) have been described. The authors concluded that energy flux densities of over 0.28 $\mathrm{mJ} / \mathrm{mm}^{2}$ should be avoided in the treatment of tendon disorders. Several morphological alterations within tendon and paratenon and further detrimental 
tissue effects depending on the shock wave intensity were observed on rabbit as well as rat models respectively ${ }^{14,15}$.

On the contrary, the first work describing shock waves benefits on tendon tissue was conducted in 2001 by Orhan et al. ${ }^{16}$, who adopted a rat model to investigate histopathological and biochemical effects of ESWT on experimentally induced full cut of Achilles tendons followed by suture. They found better healing with decrease in fibrillar disorganization in the ESWT group as compared to the control group at 3 weeks, and increased hydroxyproline (HYP) formation in the ESWT group on days 3 and 9 , which may be considered an indirect sign of collagen synthesis.

Moreover, in a histological and immunohistochemical study on rabbit model, Wang et al. ${ }^{1}$ demonstrated that ESWT induces neovascularisation, as confirmed by early release of angiogenesis-related markers, including Vascular Endothelial Growth Factor (VEGF), endothelial nitric oxide synthase (eNOS) and proliferating cell nuclear antigen (PCNA) at the Achilles tendon-bone junction. Therefore, these authors suggested that neovascularization can improve blood supply and induce tissue regeneration at the tendonbone junction. In 2006, also Kersh et al. confirmed a significant increased neovascularisation in ESWT exposed tendons, compared with untreated tendons, in horses with collagenase induced superficial digital flexor tendonitis, either early or 4 weeks after the treatment ${ }^{17}$.

After wards, Chen et al. published in 2004 an interesting study on a rat model ${ }^{5}$, investigating the effect of $0.16 \mathrm{~mJ} / \mathrm{mm}^{2}$ shock waves (at different impulses) on the healing of a collagenase-induced Achilles tendinopathy. The authors evaluated biological (at molecular level and for glycosaminoglycan and HYP content) and biomechanical (load to failure and stiffness) properties of the healing tendons and performed histological assessments. They reported that 200 impulses of shock waves determine a renewal of healthy parameters, including the resolution of oedema, swelling and inflammatory cell infiltration in the healing tendons. During the healing period, progressive tissue regeneration and cell proliferation -associated with early TGF- $\beta 1$ followed by persistent IGF-I expression- were observed. In fact, these growth factors have been found to up-regulate extracellular matrix biosynthesis of tenocytes; in particular TGF- $\beta 1$ is considered a potent inhibitor of macrophages-induced extracellular matrix degradation and inflammation during the healing of a wound. On the contrary, they reported that more than 200 impulses induced inhibitory effects of tendon repair.

Similar results - at histological, biomechanical and biochemical level - were obtained on a rabbit model of a collagenase-induced patellar tendinopathy ${ }^{2}$, using ESWT at 1500 shots at $0.29 \mathrm{~mJ} / \mathrm{mm}^{2}$. Already at 4 weeks after ESWT, they reported a significant increase of the ultimate tensile load, associated with higher HYP and pyridinoline concentrations, which represents a reliable index of newly formed and ma- ture collagen, respectively. Interestingly, at the 4th week, immature fibroblast-like tenocytes embedded in a pale eosinophilic matrix were observed in treated tendons, whereas denser and plump fibroblastlike cells in disarrayed collagen were detectable in control samples. The authors suggested that ESWT might enhance the healing of collagenase-induced patellar tendinopathy by increasing biomechanical tissutal properties and inducing higher collagen concentration.

In a pony model study ${ }^{6}$, increased glycosaminoglycan and total ECM protein syntheses -3 hours after shock waves exposure - were detected. Such results may be considered indicative for an early stimulating ESWT effect on cell metabolism, which may accelerate the healing process in injured tendons. Moreover, these authors described -3 hours following exposure - disorganisation of matrix structure with degradation of normal collagen fiber, which were detectable up to 6 weeks.

In a more recent report ${ }^{18}$, the same group observed 6 weeks after ESWT exposure - an up-regulation of MMP14 and COL1 gene expression, probably ascribable to a shock waves-induced repair phenomenon. In fact, MMP-14 should play an important role during the remodelling phase of tendon healing, generally occurring several weeks after the original trauma. However, considering the early collagen-degradation effects on exposed tissues, the shock wave exposure on non injured tissue is matter of debate, and the authors stated that it may be advisable to consider a temporary restriction of the physical activities in recently treated patients.

In 2011 Zhang et al. ${ }^{19}$ demonstrated, in a rat model study, that ESWT stimulates endogenous lubricin production in tendons and septa exposed. Clinically, an increased lubricin deposition in tendons and septa following ESWT may contribute to the beneficial effects of ESWT. This may be probably obtained by facilitating movement among gross structures, as well as among collagen fascicles, which results in a decrease of wear and tear in tissues treated and relief from symptoms and pain. AA hypothesized that ESWT could determine the expression of appropriate cytokines (TGF- $\beta$ and BMP-7) in fibroblast-like cells, which in turn increases lubricin expression in the local tissues.

In 2012 Yoo at al. ${ }^{20}$ published an interesting study in a rat model, using histopathology and atomic force microscopy (AFM), to quantitatively evaluate - the effects of four sessions of 1000 impulses at 0.085 $\mathrm{mJ} / \mathrm{mm}^{2}$ of ESWT on the healing of a collagenase induced Achilles tendinitis (CIAT). Progressive changes in nanostructure - including the fibrillary diameter and D-periodicity - and biomechanical properties - such as the fibrillary adhesion forces - over a 5-week period were observed. In particular, the authors demonstrated that ESWT may promote the healing response and reduce the healing period in the rat CIAT. In fact, early vascularity, fibroblastic activity, lymphocyte and plasma cell infiltration and disorganization of the fibers, associated with a progres- 
sive improvement in collagen fibrils nanostructure and mechanical properties were detected only in the ESWT group.

\section{Experimental researches with in vitro cell line systems}

Chao et al. ${ }^{7}$ evaluated in 2008 the effects of ESWT on primary cultured rat tenocytes harvested from Achilles tendons exposed to two different energy levels $\left(0.36,0.68 \mathrm{~mJ} / \mathrm{mm}^{2}\right)$ and four different shock wave amounts (50, 100, 250 and 500 impulses). 24, 48 and 96 hours following the treatment, cell viability was evaluated besides biochemical activity and cellular gene expression. A dose-dependent impairment of cell viability associated with significant inhibitory effects at higher intensity of shock waves was reported. On the contrary, an optimal dosage of ESWT can stimulate a significant eNOS release, tenocyte proliferation and collagen type I and III synthesis mediated by up-regulation of PCNA and TGF- $\beta 1$ gene expression. These results were confirmed by Berta et al. ${ }^{8}$ who exposed normal human fibroblast cell line (NHDF-12519) in suspension to different impulses and energies of shock waves. They reported a dosedependent destructive effect of ESWT, but also a dose-dependent stimulatory outcome on cell proliferation. They also described significant increase of $\mathrm{mR}$ NA expression for TGF- $\beta 1$ and for collagen type I and III that are consistent with activation and acceleration of the healing process.

Recently, Raabe et al. ${ }^{21}$ investigated ESWT effects on the viability, proliferation, and differentiation capacity of adipose tissue-derived mesenchymal stem cells (ASCs) in a equine model, confirming the possibility to use such approach in order to optimize healing processes of tendons. In particular an ESWT-induced increase of cell proliferation and viability was confirmed by the expression of the Ki67 marker as well as by the results of the MTT [3-(4,5-dimethylthiazol-2-yl)-2,5-diphenyltetrazolium bromide] viability assay. Interestingly the possible mitogenic shock waves-mediated effects were also appropriately evaluated through the p-Erk1-2 enhancement. Their expression was significantly increased by such exposure, because it is involved in the regulation of cell growth and differentiation. Furthermore, the authors suggest that a previous stem cells ESWT stimulation may positively affect the efficiency of transplantation procedures.

\section{Experimental studies on primary cultured human tenocytes}

At present, to our knowledge, only three experimental studies have been published to evaluate the effects of ESWT on primary cultures of human tenocytes, two of which were conducted by our study group. In particular, in our first study we assessed shock waves on a model of primary cultured human teno- cytes derived from healthy semitendinosus tendon of patients undergoing arthroscopic anterior cruciate ligament reconstruction ${ }^{11}$. A focused electromagnetic shock-wave device (Modulith SLK, STORZ Medical, Switzerland) was used and the dose of 1000 impulses at $0.14 \mathrm{~mJ} / \mathrm{mm}^{2}$ was accurately selected, because it showed satisfactory results in vivo ${ }^{22,23}$ and did not significantly interfere in vitro with the common parameters of cell viability and cytoskeleton organization $^{11}$. Nevertheless, in the only study preceding ours on ESWT effects on human cultured tenocytes - derived from diseased Achilles and healthy flexor hallucis longus tendon tissues -, a similar amount of energy was applied to the cells ${ }^{24}$. In this study, Han et al. demonstrated higher levels of several metalloproteinases (MMPs) and interleukins (ILs) in human tendinopathy-affected tenocytes and their significant decrease after shock waves exposure. However, the cultured cells were analysed only at short term, at 72 hours after shock waves exposure, whereas it is generally accepted that the clinical benefits are detected no earlier that 2 weeks following the treatment 25,26 . Hence, in our aforementioned study, we investigated the tenocyte behaviour during a 12-day period following the exposure ${ }^{11}$. Because one of the possible ESWT-mediated mechanisms of action involves the tendon healing, we focused on the tenocyte morphology, differentiation, proliferation, migration and collagen synthesis, all of which are potentially able to influence the tissue repair process.

In this initial study, we performed cultures derived exclusively from healthy tendon, which might show different functional activities compared to ruptured tendons. To overcome this limitation, in our second study we compared cultures of human tenocytes explanted by ruptured Achilles (AT) versus healthy semitendinosus (ST) tendons, both at very early passage (P1) and following (or not) shock waves exposure ${ }^{12}$.

As previously reported in a model of animal tendonderived cells ${ }^{27}$, results of our studies confirmed that a phenotypic drift progressively occurs through following passages, showing that a first group of classically elongated fibroblast-like cells became prevalent on a second group of ovoid tenoblast-like cells. Our observations supported the view that untreated human cultured tenocytes could be mainly considered as differentiated elements ${ }^{11,28,29}$, showing an unstable morphology with a progressive tendency to dedifferentiate in vitro ${ }^{30}, 31$. Interestingly, ESWT significantly impaired such natural trend of human tenocytes to dedifferentiate in vitro, suggesting that the morphological changes observed in vitro may be responsible for the matrix alterations showed in tendinopathic tendons in vivo $^{31,32}$.

Thence, collagen synthesis and cell proliferation of treated compared to control tendon-derived-cells were investigated. As expected, shock waves exposure significantly increased collagen production in vitro, mainly belonging to type I, which is crucial during the later events of healing ${ }^{11,28}$. Similarly, the analysis of Ki67 and 5-bromo-2'-deoxyuridine (BrdU) proliferation markers confirmed a significant ESWT-mediated 
growth stimulation, becoming more conspicuous at late passage cultures. Then we compared the growth rate - on cell cycle distribution by cytofluorimetry - of healthy versus ruptured tendon-derived cells, showing that before treatment the two groups were characterized by similar percentages of proliferative cells. Interestingly, biological effects of such treatment was more evident in tenocytes derived from ruptured than from healthy tendons, as ascertained by an increase of 1.75 folds in the ratio of proliferative activity ${ }^{11,12}$.

A further observation corroborating the evidence of a specific ESWT-mediated effect on tenocytes explanted from ruptured tendon was shown by a scratch migration assay, which demonstrated that the exposure induces - mainly in those samples - a typical migratory phenotype as well as a significant increase of cell motility. Finally, at molecular level, we analysed by real-time PCR cultured cells derived either from healthy or ruptured tendons, in order to better characterize our tenocytes and detect possible changes induced by ESWT in tendon specific gene marker expression, including Collagens I and III (Col I-III), Scleraxis (Scx), Tenomodulin ( $\mathrm{Tnm})$ and Tenascin-C (TnC). Tendon-specific markers were simultaneously detected in all our cultures, confirming the quality of our cell model, independently from the source of the explants $^{12}$. Interestingly, the expression of Scx, a typical early differentiative marker ${ }^{33}$, was significantly decreased after shock waves exposure in ruptured tendon-derived cultures, whereas the later differentiative marker Tnm was not yet modified ${ }^{12}$. From all these recent results, we may state that ruptured compared to healthy tendon-derived cell cultures represent a more convincing model to better evaluate the effects of ESWT, because this treatment is obviously performed in vivo only on pathological tendons. Our data may also suggest a possible activation in ruptured tendon of an early differentiative program mediated by shock waves exposure, further favouring the repair of the tendon tissue.

The primary human tendon cell cultures have been conducted in normoxia. Using hyperoxic $(90 \%, 02)$ compared to normoxic $(21 \%, 02)$ conditions for culturing cells can potentially influences the shock waves-induced effects, for example through an abnormal extracellular matrix deposition ${ }^{34}$. However, at the present time, no data are available on the effects of shock wave treatment in tenocyte cultures under hyperoxic conditions.

Finally, the main limitation of in vitro depends by the fact that these data could never be used to provide impeccable conclusions regarding in vivo conditions, particularly in a microenvironment characterized by the presence of a complex network of molecules able to trigger different kinds of events. A further limitation of our in vitro model was that the primary cultured human tenocytes were explanted from two different sources (i.e. Semitendinosus and Achilles tendons) and, even though they showed a simultaneous expression of the typical tenocyte markers, we can't exclude that human tendon-derived cultures explanted from other sources could exhibit different gene expression signatures, determining different cell behaviour.

\section{Conclusion}

The in vitro studies on the ESWT effects in tendon models are shedding light on the possible mechanisms of action of such treatment, although the experiments in vitro cannot be directly generalised to the in vivo conditions.

The majority of the studies have shown a dose-dependent destructive effect of ESWT, but they provided also evidence that an optimal dosage of ESWT determines a stimulatory effect on cell proliferation, as well as the activation and enhancement of healing process. In fact, taken together, the morphological changes, proliferation and motility of treated cells, functional outcome on neovascularization and collagen synthesis, as well as the expression of differentiation critical genes suggest that ESWT may be able to increase tendon healing. Nevertheless, we are not yet able to define how, in vivo, the ESWT acts on exposed tissues. They may provide mechanical and biological stimuli determining the activation of a complex network of molecules, including a large panel of cytokines and metalloproteinases ${ }^{24,}$ 35-37.

In conclusion, although the mechanisms of action of shock waves are still not fully clear yet, the surprising clinical benefits induced by ESWT resulted in a continuous increase of request for such treatment, which is recently starting to be used also in regenerative medicine. Nevertheless, further experimental studies are needed to better elucidate the exact mechanisms triggered by ESWT in healthy and pathological human tissues ${ }^{38}$.

\section{Acknowledgments}

The authors thank Dr. Francesca Terranova, Università degli Studi di Roma "Sapienza", for English language revision in preparing the manuscript.

\section{References}

1. Wang CJ, Wang FS, Yang KD, et al. Shock wave therapy induces neovascularization at the tendon-bone junction. A study in rabbits. J Orthop Res. 2003;21:984-989.

2. Hsu RW, Hsu WH, Tai CL, Lee KF. Effect of shock-wave therapy on patellar tendinopathy in a rabbit model. J Orthop Res. 2004;22:221-227.

3. Notarnicola A, Moretti B. The biological effects of extracorporeal shock wave therapy (eswt) on tendon tissue. Muscles Ligaments Tendons J. 2012;2:33-37.

4. Frairia R, Berta L. Biological effects of extracorporeal shock waves on fibroblasts. A review. Muscles Ligaments Tendons J. 2011;1:138-147.

5. Chen YJ, Wang CJ, Yang KD, et al. Extracorporeal shock waves promote healing of collagenase-induced Achilles tendinitis and increase TGF-beta1 and IGF-I expression. J Orthop Res. 2004;22:854-861. 
6. Bosch G, Lin YL, van Schie HT, van De Lest CH, Barneveld A, van Weeren PR. Effect of extracorporeal shock wave therapy on the biochemical composition and metabolic activity of tenocytes in normal tendinous structures in ponies. Equine Vet $\mathrm{J}$. 2007;39:226-231.

7. Chao YH, Tsuang YH, Sun JS, et al. Effects of shock waves on tenocyte proliferation and extracellular matrix metabolism. UItrasound Med Biol. 2008;34:841-852.

8. Berta L, Fazzari A, Ficco AM, Enrica PM, Catalano MG, Frairia R. Extracorporeal shock waves enhance normal fibroblast proliferation in vitro and activate mRNA expression for TGF-beta1 and for collagen types I and III. Acta Orthop. 2009;80:612-617.

9. Schulze-Tanzil G, Mobasheri A, Clegg PD, Sendzik J, John T, Shakibaei M. Cultivation of human tenocytes in high-density culture. Histochem Cell Biol. 2004;122:219-228.

10. Buhrmann $C$, Mobasheri A, Busch F, et al. Curcumin modulates nuclear factor kappaB (NF-kappaB)-mediated inflammation in human tenocytes in vitro: role of the phosphatidylinositol 3-kinase/Akt pathway. J Biol Chem. 2011;286:2855628566.

11. Vetrano M, d'Alessandro F, Torrisi MR, Ferretti A, Vulpiani MC, Visco V. Extracorporeal shock wave therapy promotes cell proliferation and collagen synthesis of primary cultured human tenocytes. Knee Surg Sports Traumatol Arthrosc. 2011:19:2159-2168.

12. Leone L, Vetrano M, Ranieri D. Extracorporeal Shock Wave Treatment (ESWT) improves in vitro functional activities of ruptured human tendon-derived tenocytes. PLoS One. 2012;7:e49759.

13. Rompe JD, Kirkpatrick CJ, Kullmer K, Schwitalle M, Krischek O. Dose-related effects of shock waves on rabbit tendo Achillis. A sonographic and histological study. J Bone Joint Surg Br. 1998;80:546-552.

14. Maier M, Tischer T, Milz S, et al. Dose-related effects of extracorporeal shock waves on rabbit quadriceps tendon integrity. Arch Orthop Trauma Surg. 2002;122:436-441.

15. Orhan Z, Cam K, Alper M, Ozturan K. The effects of extracorporeal shock waves on the rat Achilles tendon: is there a critical dose for tissue injury? Arch Orthop Trauma Surg. 2004; 124:631-635.

16. Orhan Z, Alper M, Akman $\mathrm{Y}$, Yavuz O, Yalciner A. An experimental study on the application of extracorporeal shock waves in the treatment of tendon injuries: preliminary report. J Orthop Sci. 2001;6:566-570.

17. Kersh KD, McClure SR, Van Sickle D, Evans RB. The evaluation of extracorporeal shock wave therapy on collagenase induced superficial digital flexor tendonitis. Vet Comp Orthop Traumatol. 2006;19(2):99-105.

18. Bosch $G$, de Mos M, van Binsbergen R, van Schie $H T$, van de Lest $\mathrm{CH}$, van Weeren PR. The effect of focused extracorporeal shock wave therapy on collagen matrix and gene expression in normal tendons and ligaments. Equine Vet J. 2009;41:335-341.

19. Zhang D, Kearney CJ, Cheriyan T, Schmid TM, Spector M. Extracorporeal shockwave-induced expression of lubricin in tendons and septa. Cell Tissue Res. 2011;346(2):255-256.

20. Yoo SD, Choi S, Lee GJ, Chon J, Jeong YS, Park HK, Kim HS. Effects of extracorporeal shockwave therapy on nanostructural and biomechanical responses in the collagenaseinduced Achilles tendinitis animal model. Lasers Med Sci. 2012;27(6):1195-1204
21. Raabe O, Shell K, Goessl A, et al. Effect of extracorporeal shock wave on proliferation and differentiation of equine adipose tissue-derived mesenchymal stem cells in vitro. Am J Stem Cells. 2013;2(1):62-73.

22. Vulpiani MC, Trischitta D, Trovato P, Vetrano M, Ferretti A. Extracorporeal shockwave therapy (ESWT) in Achilles tendinopathy. A long-term follow-up observational study. J Sports Med Phys Fitness. 2009;49:171-176.

23. Vulpiani MC, Vetrano M, Savoia V, Di Pangrazio E, Trischitta $D$, Ferretti A. Jumper's knee treatment with extracorporeal shock wave therapy: a long-term follow-up observational study. J Sports Med Phys Fitness. 2007;47:323-328.

24. Han SH, Lee JW, Guyton GP, Parks BG, Courneya JP, Schon LC. J. Leonard Goldner Award 2008. Effect of extracorporeal shock wave therapy on cultured tenocytes. Foot Ankle Int. 2009;30:93-98.

25. Speed CA, Richards C, Nichols D, Burnet S, Wies JT, Humphreys $\mathrm{H}$, et al. Extracorporeal shock-wave therapy for tendonitis of the rotator cuff. A double-blind, randomised, controlled trial. J Bone Joint Surg Br. 2002; 84:509-512.

26. Rompe JD, Maffulli N. Repetitive shock wave therapy for lateral elbow tendinopathy (tennis elbow): a systematic and qualitative analysis. Br Med Bull. 2007;83:355-378.

27. Yao L, Bestwick CS, Bestwick LA, Maffulli N, Aspden RM Phenotypic drift in human tenocyte culture. Tissue Eng. 2006;12:1843-1849.

28. Bernard-Beaubois K, Hecquet C, Houcine O, Hayem G, Adolphe M. Culture and characterization of juvenile rabbit tenocytes. Cell Biol Toxicol. 1997;13:103-113.

29. Kannus P. Structure of the tendon connective tissue. Scand Med Sci Sports.2000;10:312-320.

30. Stoll C, John T, Endres M, et al. Extracellular matrix expression of human tenocytes in three-dimensional air-liquid and PLGA cultures compared with tendon tissue: implications for tendon tissue engineering. J Orthop Res. 2010;28:1170-1177.

31. Maffulli N, Ewen SW, Waterston SW, Reaper J, Barrass V. Tenocytes from ruptured and tendinopathic achilles tendons produce greater quantities of type III collagen than tenocytes from normal achilles tendons. An in vitro model of human tendon healing. Am J Sports Med. 2000;28:499-505.

32. Denaro V, Ruzzini L, Longo UG, et al. Effect of dihydrotestosterone on cultured human tenocytes from intact supraspinatus tendon. Knee Surg Sports Traumatol Arthrosc. 2010;18:971976.

33. Shukunami C, Takimoto A, Oro M, Hiraki Y. Scleraxis positively regulates the expression of tenomodulin, a differentiation marker of tenocytes. Dev Biol. 2006;298:234-247.

34. Rebours V, Albuquerque M, Sauvanet A, Ruszniewski P, Lévi P, Paradis V, Bedossa P, Couvelard A. Hypoxia pathways and cellular stress activate pancreatic stellate cells: development of an organotypic culture model of thick slices of normal human pancreas. PLoS One. 2013; 9: e76229.

35. Oliva F, Barisani D, Grasso A, Maffulli N. Gene expression analysis in calcific tendinopathy of the rotator cuff. Eur Cell Mater. 2011;21:548-557.

36. Del Buono A, Oliva F, Longo UG, et al. Metalloproteases and rotator cuff disease. J Shoulder Elbow Surg. 2012;21:200-208.

37. Del Buono A, Oliva F, Osti L, Maffulli N. Metalloproteases and tendinopathy. Muscles Ligaments Tendons J. 2013;3:51-57.

38. Padulo J, Oliva F, Frizziero A, Maffulli N. Muscles, Ligaments and Tendons Journal. Basic principles and recommendations in clinical and field science research. MLTJ. 2013;4:250-252. 